Ann. Zootech., I969, 18 (I), 5-I5.

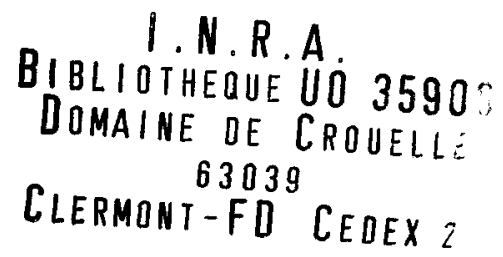

\title{
RELATIONS ENTRE L'APTITUDE A LA TRAITE DES BREBIS ET LA PERTE DE PRODUCTION LAITIÈRE CONSTATÉE AU MOMENT DU SEVRAGE
}

\author{
J. LABUSSIÈRE, P. PÉTREQUIN \\ avec la collaboration technique de J.-F. Combaud et B. Mirman \\ Laboratoire de Physiologie de la Lactation, \\ Centre national de Recherches zootechniques, 78 - Jouy-en-Josas \\ Institut national de la Recherche agronomique
}

\section{SOMMAIRE,}

Au cours de la traite mécanique, les brebis présentant un réflexe d'éjection du lait (animaux à 2 émissions, lot 2) ont une production supérieure à celles n'en possédant pas (lot I).

Au cours de la phase d'allaitement, pourtant, il n'existe pas de différence significative entre les deux lots. La chute immédiate constatée au moment du sevrage brutal à 42 jours est identique et essentiellement due à la disparition complètz du réflexe neuro-endocrinien d'éjection.

Sa réapparition progressive chez les brebis à deux émissions doit être tenue pour une grande part comme responsable de la production supérieure constatée alors chez ces animaux.

La perte de lait enregistrée entre 42 jours d'allaitement et 42 jours de traite est d'autant plus faible que les mécanismes physiologiques d'éjection évoqués par la machine sont efficaces (volumes absolu et relatif de la $2^{\mathrm{e}}$ émission importants, débit maximum élevé).

Il semble également qu'un comportement maternel très développé accroît de façon notable l'écart observé entre le dernier contrôle de tétée et celui de la première semaine de traite. Cet écart est en effet d'autant plus grand que la persistance de la production individuelle de chaque brebis en période d'allaitement est forte.

Dans aucun cas la quantité de lait recueillie à la traite n'est susceptible d'atteindre le niveau des courbes de production obtenues lors de la tétée. Des hypothèses sont formulées concernant les causes de ces phénomènes.

\section{I. - INTRODUCTION}

I. De très nombreux chercheurs ont constaté que la production laitière des brebis est fortement réduite après le sevrage des agneaux. En effet, les performances enregistrées à la traite sous-estiment d'environ $30 \mathrm{p}$. Ioo les potentialités sécrétoires extériorisées par les brebis-mères en période d'allaitement. C'est là un chiffre moyen 
qui varie suivant le nombre d'agneaux allaités, le mode de sevrage et le stade auquel celui-ci intervient. Ainsi, la confrontation des deux contrôles qui encadrent cette phase importante, conduit à une perte de $32 \mathrm{p}$. Ioo pour FINCI (I957), de II à 22 p. IOO pour GEORGIOU (I960), de 20 à 40 p. IOO pour RICORDEAU et DENAMUR (I962), de 30 à 40 p. IOO pour Folman, Vor,CANI et Exal, (I966). Des observations analogues ont été également effectuées par GAAL (I966) et Mikus (I965).

2. Lorsqu'on fait porter la comparaison non plus sur deux périodes successives de la lactation, mais sur deux lots différents d'animaux (1'un trait, l'autre tété) choisis à des stades physiologiques identiques, on constate, là aussi, que la production des brebis traites est inférieure à celle des brebis allaitantes : $40 \mathrm{p}$. Ioo pour Scheinaker (I929), 25 p. Ioo pour Markus (I962) et Eidel,Man (I963).

3. Cette supériorité de l'agneau est vraisemblablement due pour une grande part à son aptitude à vider la mamelle plus parfaitement que la machine ou la main du trayeur $\left({ }^{1}\right)$. En effet, après une traite considérée comme complète, la tétée du jeune permet encore une récupération importante de lait qui a été chiffrée à $23 \mathrm{p}$. Ioo du total par F,yal, Volcani et Sharav (I958), à 38 p. IOO par CiolCa et al. (I960), à 25 p. IOO environ par RICORDEAU et DENAMUR (I962), enfin, entre 24 et 37 p. Ioo par Folman, Volcani et Exal, (Ig66).

4. Signalons en outre que l'efficience du réflexe d'éjection est sensiblement améliorée par la seule présence de l'agneau auprès de sa mère au moment de la traite. La quantité de lait recueillie est alors supérieure (CoNsTANTINESCU et GoNdos, I958), et la vitesse à laquelle il est extrait est nettement plus rapide (RICORDEAU et DENAmur, I962).

Une étude des courbes de descente de lait dans les conditions normales de traite

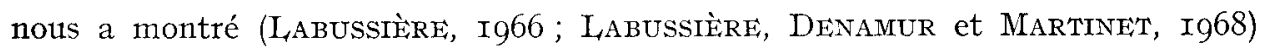
qu'il existe schématiquement deux catégories d'animaux :

a) ceux fournissant leur lait en deux émissions successives bien distinctes correspondant à l'écoulement du lait citernal puis du lait alvéolaire chassé des acini sous l'effet de l'ocytocine ;

b) ceux qui ne présentent que la première émission probablement à la suite d'une inhibition du réflexe neuro-endoctinien d'éjection dont la cause nous est encore inconnue.

Compte tenu des différents résultats qui viennent d'être exposés, on peut supposer que cette inhibition n'existe pas (ou peu) pendant la phase d'allaitement et que le comportement maternel de la brebis vis-à-vis de son agneau rend possible une extraction abondante du lait.

Selon cette hypothèse, la perte de production que l'on constate après le sevrage serait imputable :

a) surtout aux animaux à une seule émission dont 1'adaptation à la machine à traire paraît difficile ;

(1) Néanmoins cette extraction du lait par l'agneau n'est pas encore parfaite, puisqu'après la tétée une injection d'ocytocine provoque l'écoulement d'une quantité importante de lait résiduel (RICORDFAU et DENAMUR, I962). 
b) éventuellement aux brebis à 2 pics dont le réflexe d'éjection peut être moins efficient.

C'est ce que nous avons voulu vérifier au cours de l'étude qui va être exposée ci-dessous.

\section{II. - MATÉRIEL ET MÉTHODES}

\section{A. - Animaux}

Quarante-cing brebis Préalpes du Sud en première lactation (âge I an) furent utilisées pour ce travail : I9 mirent bas entre le 24 octobre et le 19 novembre 1965 , et 26 entre le 27 février et le I2 avril 1967 .

Tons les animaux allaitèrent seulement un agneau pendant 6 semaines. Le sevrage fut brutal.

La répartition en brebis à une et deux émissions fut effectuée a posteriori, après examen des courbes de descente de lait.

\section{B. - Technique de traite}

La traite eut lieu 2 fois par jour à 6 heures et 16 heures, sur un "carroussel "à 32 places équipé d'une machine Alfa-Laval dont les caractéristiques sont les suivantes :

$$
\begin{aligned}
& \text { vide }: 33 \mathrm{~cm} / \mathrm{Hg} \\
& \text { I } 80 \text { pulsations } / \text { minute } \\
& \text { rapport succion/massage : } \mathrm{I} / \mathrm{I}
\end{aligned}
$$

Les gobelets pesant environ $240 \mathrm{~g}$ furent toujours posés immédiatement, sans massage ni préparation préalable cle la mamelle. Ia finition de la traite fut assurée par un égouttage-machine, puis, 2 minutes environ après la dépose de la griffe, par un égouttage manuel ou "repasse".

$$
\text { C. - Contrôles }
$$

I. Production laitière.

a) Pendant la période d'allaitement.

La production laitière fut estimée par pesée de l'agneau avant et après la tétée selon la méthode proposée par RiCORDEAU, BOCCARD et DENAMUR (I960), et que l'on peut résumer brièvement. Ces pesées eurent lieu à 7 heures, 9 heures, I I heures, I 3 heures, I 5 heures, I 7 heures et ig heures, la première n'étant pas retenue pour le calcul final puisque représentant le produit de sécrétion d'un intervalle de temps inconnu. Le total des 6 autres tétées fut doublé pour obtenir le rendement en 24 heures.

\section{b) Pendant la période de traite.}

Pendant les 48 premiers jours qui suivirent le sevrage, la quantité de lait fournie par chaque brebis fut mesurée à chaque traite avec une précision voisine de 10 millilitres.

2. Cinétique de descente de lait.

L'enregistrement fut effectué à l'aide d'un suiveur de niveau (LABUssì̀re et MARTineT, 1964), et d'une chaîne de mesure Beckman comprenant un voltmètre digital et une imprimante.

La plupart des animaux furent contrôlés pendant les Io premiers jours de traite ${ }^{1}$ ), puis ensuite

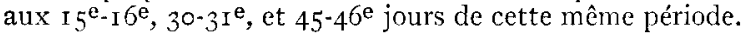

(1) Certaines brebis ayant mis bas en 1965 ne subirent ces contrôles que pendant les 5 premiers jours puis ensuite toutes les 2 semaines à plusieurs traites consécutives. 
D. - Expression des résultats

1. Pertes consécuticies au sezrage.

a) Perte du premier jour $\left(\mathrm{P}_{1}\right)$.

La production laitière du premier jour de traite est exprimée en p. roo de celle du demier contrôle d'allaitement. La perte est la différence entre ce rapport et ı00 P. I00.

b) Perte de la première simaine $\left(\mathrm{P}_{-}\right)$.

Le mode d'estimation est identique, mais on remplace la donnée du premier jour par celle de la moyenne quotidienne de la première semaine.

c) Perte sur 42 jours $\left(\mathrm{P}_{42}\right)$.

La production pendant les 42 premiers jours de traite a été rapportée (en p. I00) i celle évaluée sur un laps de temps équivalent pendant la phase d'allaitement (1). Comme précédenment, la perte représente le complément à roo de la valeur relative ainsi calculée.

\section{Criteres d'appréciation de l'aptitude à la traite.}

Parmi les critères définis antérieurement (LAbLssitre, ig66), nous arons retenu ceux qui permettent d'estimer l'importance du rélexe d'éjection du lizit qui, lorsclu'il est intense. se manifeste sous forme d'une deuxième émission dont :

- Les volumes absolu et relatif sont grands.

- Le débit maximum élevé.

- Je temps de latence très court.

\section{III. - RÉSULTATS}

\section{A. - Classement des brebis à une et deux émissions}

A l'exception de 4 animaux chez lesquels il apparaît rapidement, le réflexe d'éjection du lait n'est jamais présent pendant les Io premiers jours de traite. Ce n'est qu'à partir du I $5^{\mathrm{e}}$ jour et surtout du $30^{\mathrm{e}}$ que la distinction entre animaux à une (lot I) et deux émissions (lot 2) devient possible.

Nous avons pu alors établir la répartition suivante :

lot $\mathrm{I}: 22$ brebis

lot $2: 23$ brebis

\section{B. - Production laitière pendant les phases successives d'allaitement et de traite}

I,es courbes moyennes de lactation sont présentées sur la figure I.

En période d'allaitement, sur aucun des 6 contrôles hebdomadaires de pesée il n'existe de différence significative entre les lots i et 2 . De plus, la chute constatée au moment du sevrage est identicue pour les 2 groupes d'animaux.

Le premier jour de traite, les brebis à une émission produisent $6 \mathrm{r}, 3$ 1). Ioo de ce que tétaient les agneaux lors du dernier contrôle poncléral (soit une perte de 38,7 p. I00). Cette perte est de $36,6 \mathrm{p}$. Ioo pour les brebis à deux émissions.

Dans les deux cas, la production ne diffère pas penclant les 4 jours qui suivent le sevrage. Elle devient ensuite statistiquement plus élevée pour les animaux du lot 2 ,

(1) Ia quantité totale de lait tété par l’agneau en 42 jours a été estimée à partir de 6 contrôles hebdomadaires par la méthode Iileischman. 
l'écart continuant à s'accroître pour devenir hautement significatif à partir dı I $2^{\mathrm{e}}$ jour.

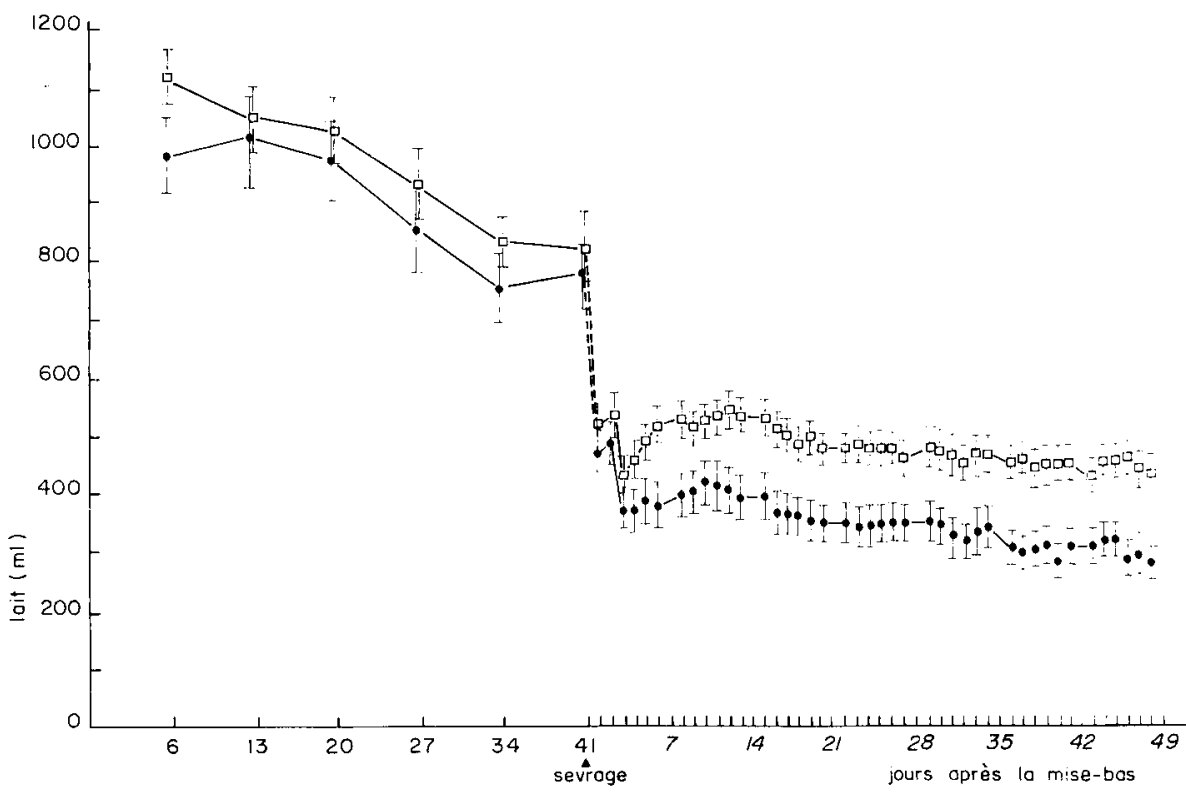

Fig. I. - Production laiticre des brebis à une et deux émissions pendant les phases successives d'allaitement et de traite

$-\square-\square-\square-$ brebis à 2 émissions

$\ldots-\ldots$ brebis à 1 émission

Ces résultats sont également illustrés d'une façon différente au tableau I.

\section{TABLEAU I}

Production laitière journalière des deux lots d'animaux pendant les deux périodes successizes d'allaitement et de traite

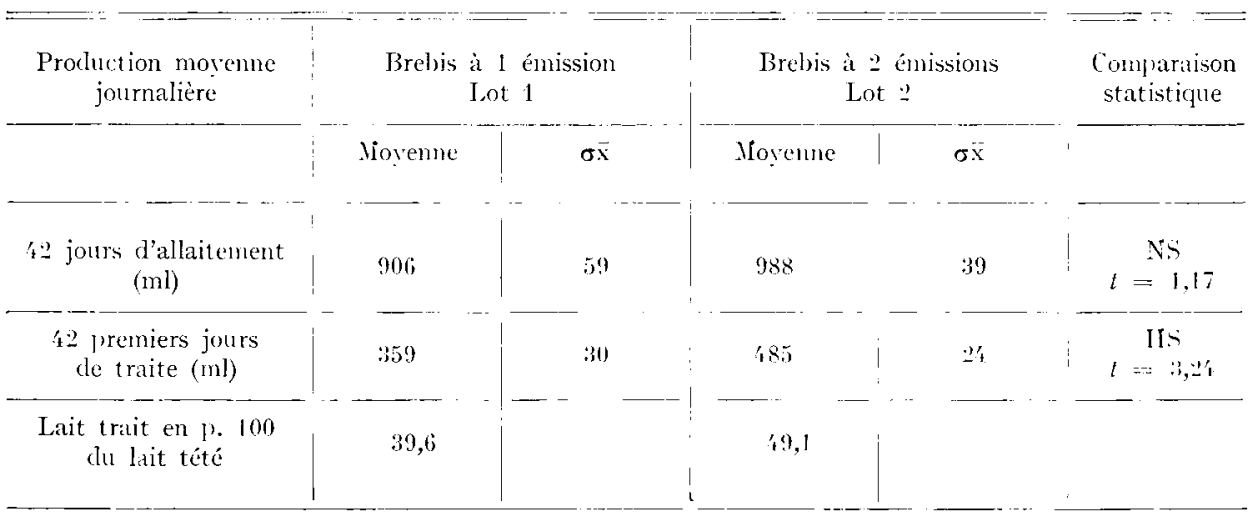

$\sigma \overline{\mathrm{x}}=$ écart-type de la moyenne. $\quad X S=$ non significatif. $\quad$ IRS $=$ hatutement significatif. 


\section{C. - Relation entre la perte de production et différents critères de traite}

Afin de mieux connaître les phénomènes qui conditionnent la perte de production au moment du sevrage, nous avons étudié les relations entre celle-ci et différents critères mesurés : en période de traite et d'allaitement.

Les résultats obtenus sont présentés au tableau 2 et sur les figures 2,3 et 4 .

TABLEAU 2

Corrélation entre différents paramètres de traite

\begin{tabular}{|c|c|c|c|c|}
\hline & $\begin{array}{l}\text { 1'erte sur } \\
42 \text { jours } \\
P_{42}\end{array}$ & $\begin{array}{l}\text { Perte de la } \\
1^{\text {re semaine }} \\
\mathbf{P}_{\mathrm{T}}\end{array}$ & $\begin{array}{c}\text { Perte dis } \\
\text { der jour } \\
\mathrm{P}_{1}\end{array}$ & $\begin{array}{l}\text { Moyenne } \\
\text { joumalière } \\
\text { allait muent }\end{array}$ \\
\hline $\begin{array}{l}\text { Volunie absolu } \\
\text { de la le émission }\left(^{1}\right)\end{array}$ & $-0,59^{* *}$ & $+0,20$ & $-0,06$ & $+01,65 * *$ \\
\hline $\begin{array}{l}\text { Volume relatif } \\
\text { de la ze emission (1) }\end{array}$ & $-0,58^{* *}$ & $+0,19$ & $-0,06$ & $+0,19^{* *}$ \\
\hline $\begin{array}{l}\text { 'leulus de latence } \\
\text { du rétlexe (1) }\end{array}$ & $+0,{ }^{\prime} 0^{*}$ & - 0,27 & $-0,1_{t}^{\prime}$ & $-0,3: 3$ \\
\hline $\begin{array}{l}\text { Débit maximum } \\
\text { de la ı̀e émission }\left({ }^{1}\right)\end{array}$ & $-0,6 \geqslant * *$ & $+0,: 1$ & $-0,06$ & $+0,57 * *$ \\
\hline $\begin{array}{l}\text { Moseme joumaliere } \\
\text { allaitement }\end{array}$ & $\begin{array}{l}-0,1 ; \text { lot }: \\
-0,21 \text { lot } 1\end{array}$ & $\begin{array}{l}+0,16 \text { lot } 2 \\
+0,16 \text { lot } 1\end{array}$ & $\begin{array}{l}0,20 \text { lot } 2 \\
-0,09 \text { lot } 1\end{array}$ & \\
\hline $\begin{array}{c}\text { Moveme journalière } \\
\text { traite }\end{array}$ & $\begin{array}{l}-0,6^{\prime} * * \text { lot }= \\
-0,7^{* *} \text { lot } 1\end{array}$ & $\begin{array}{r}-0,01 \text { lot } 2 \\
-0,33 \text { lot } 1\end{array}$ & $\begin{array}{l}-0,30 \text { lot } 2 \\
-0,2 \text { it } \\
-0 t\end{array}$ & $\begin{array}{l}+0,85^{* *} \text { lot } 2 \\
+0,66^{* *} \text { lot } 1 \\
+0,72^{* *} \text { pour } \\
\text { l'ensemble des lots }\end{array}$ \\
\hline $\begin{array}{c}\text { Coetficiut de persistance } \\
\text { :llatement }\left({ }^{*}\right)\end{array}$ & $\begin{array}{l}-0,05 \\
\text { ensemble } \\
\text { des } 2 \text { lots }\end{array}$ & $\begin{array}{l}+0,5 * * \text { lot } 2 \\
+1-0,12 \text { lot } 1 \\
+0,31 * \text { pour } \\
\text { l'ensemble } \\
\text { des } 2 \text { lots }\end{array}$ & $\begin{array}{l}+0,20 \\
\text { ensemble } \\
\text { des lots }\end{array}$ & \\
\hline
\end{tabular}

* Signilicatif (j p 100).

** ITautement significatif (1 p. 100).

(1) Nous arons choisi les données moyennes enregistrées les $30^{\mathrm{e}}$ et $32^{\circ}$ jours après le sevrage, stade auquel les courles de descente de lait sont les plus représentatives de l'animal.

(अ) Ce critiere est probablement d'autant plus faible que le comportement maternel est peu prononcé. ll est calculé entre le for et le demier contrôle d'allikitement.

I. On remarque qu'il existe une corrélation hautement significative entre :

a) Lait tété et lait trait: Ceci est valable pour les deux types d'animaux et surtout pour les brebis à 2 pics. 'Toutefois, l'étude statistique nous a montré qu'il n'exis- 
tait pas, ni pour les coefficients de corrélation $\left(r_{2}=+0,85\right.$ et $\left.\gamma_{1}=+0,66\right)$ ni pour les pentes des droites de régression de la figure $2\left(b_{2}=+0,54\right.$ et $\left.b_{1}=+0,35\right)$, de différences entre lots.

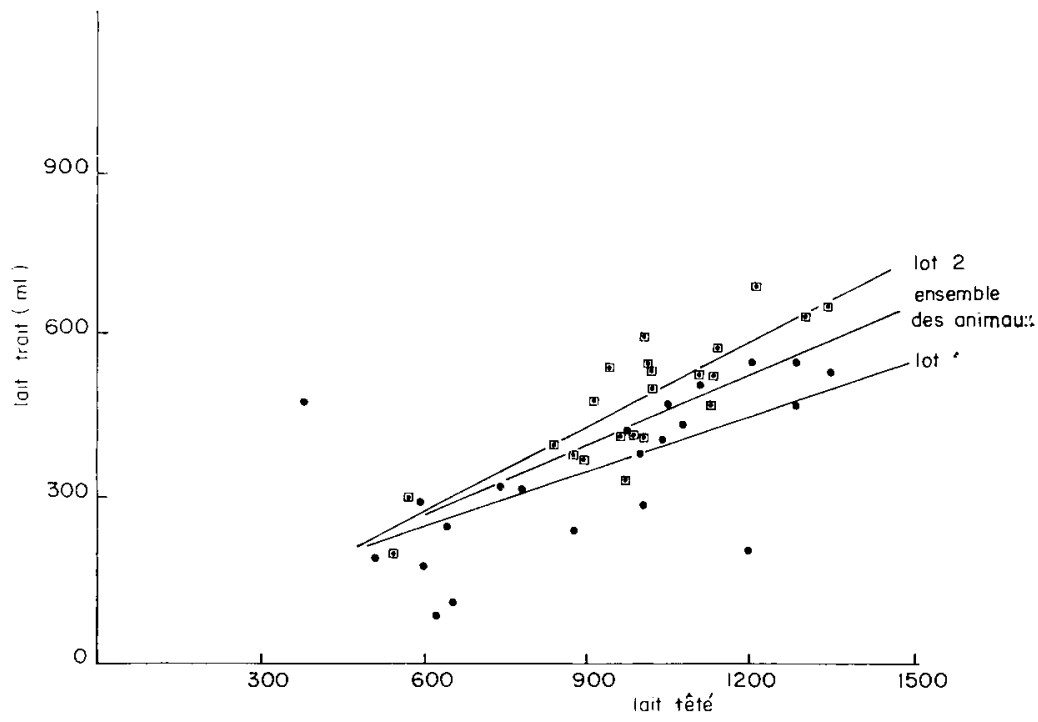

FIG. 2. - Relation enle lail tété al lait trait

-- Brcbis à une émission $y=0,346 x+53,40$

- $\square$ Brebis à deux émissions $y=0,54 \mathrm{I} x-44,47$

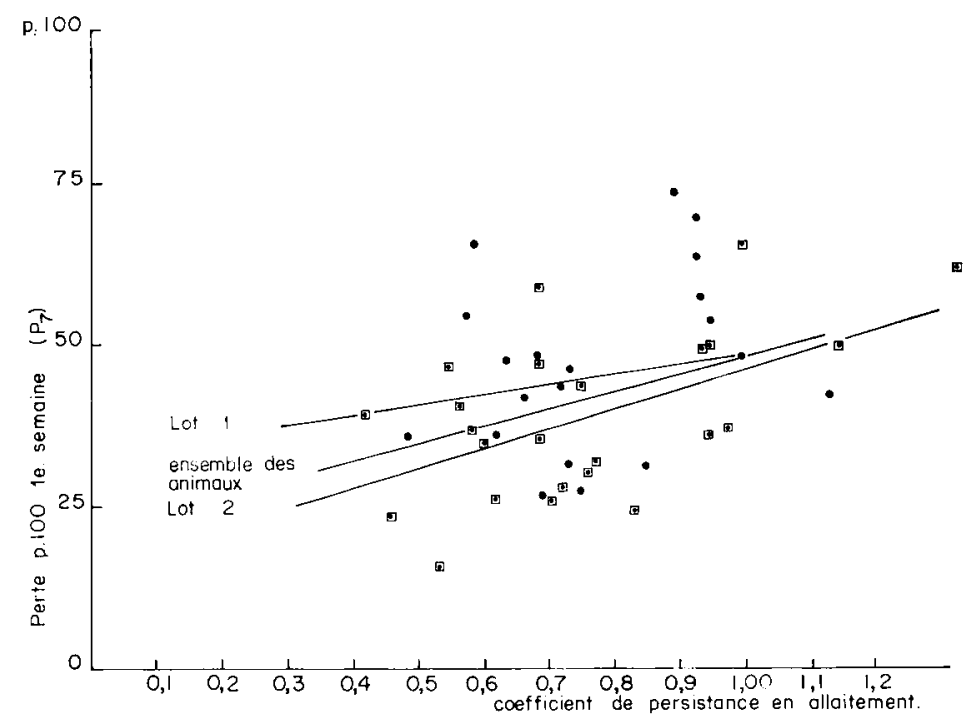

FIG. 3. - Relalion enlre la perle de la premidre semaine el la persislance en période d'allaitement

- Brebis à une émission $y=12,35 x+34,96$

- $\rightarrow$ Brebis à deux émissions $y=30,03 x+15,56$ 
b) Lait tété et quelques-uns des critères nous servant à caractériser l'ampleur de la $2^{\mathrm{e}}$ émission (volumes absolu et relatif, débit maximum).

2. Nous noterons également que la perte $P_{7}$ est étroitement et positivement reliée à la persistance en période d'allaitement, ceci étant uniquement valable pour les animaux du groupe 2 (fig. 3) (tabl. 2).

3. Enfin il apparaît que l'écart entre les deux périodes de 42 jours $\left(\mathrm{P}_{42}\right)$ est d'autant plus faible que :

a) Le réflexe d'éjection du lait provoqué par la machine semble efficient ou en d'autres termes que :

- les volumes absolu et relatif de la $2^{\mathrm{e}}$ émission sont forts : $r=-0,59$ et $-0,58$ (fig. $4 a$ et $4 b$ ) ;

- le débit de celle-ci est important : $r=-0,62$ (fig. $4 c$ );

- la latence nécessaire à la manifestation de cette $2^{\mathrm{e}}$ émission est courte : $r=+0,40$ (fig. $4 d$ ).

b) La production moyenne de lait trait est élevée ce qui s'avère particulièrement logique $(r=+0,74$ et $+0,64$ respectivement pour les lots I et 2$)$.
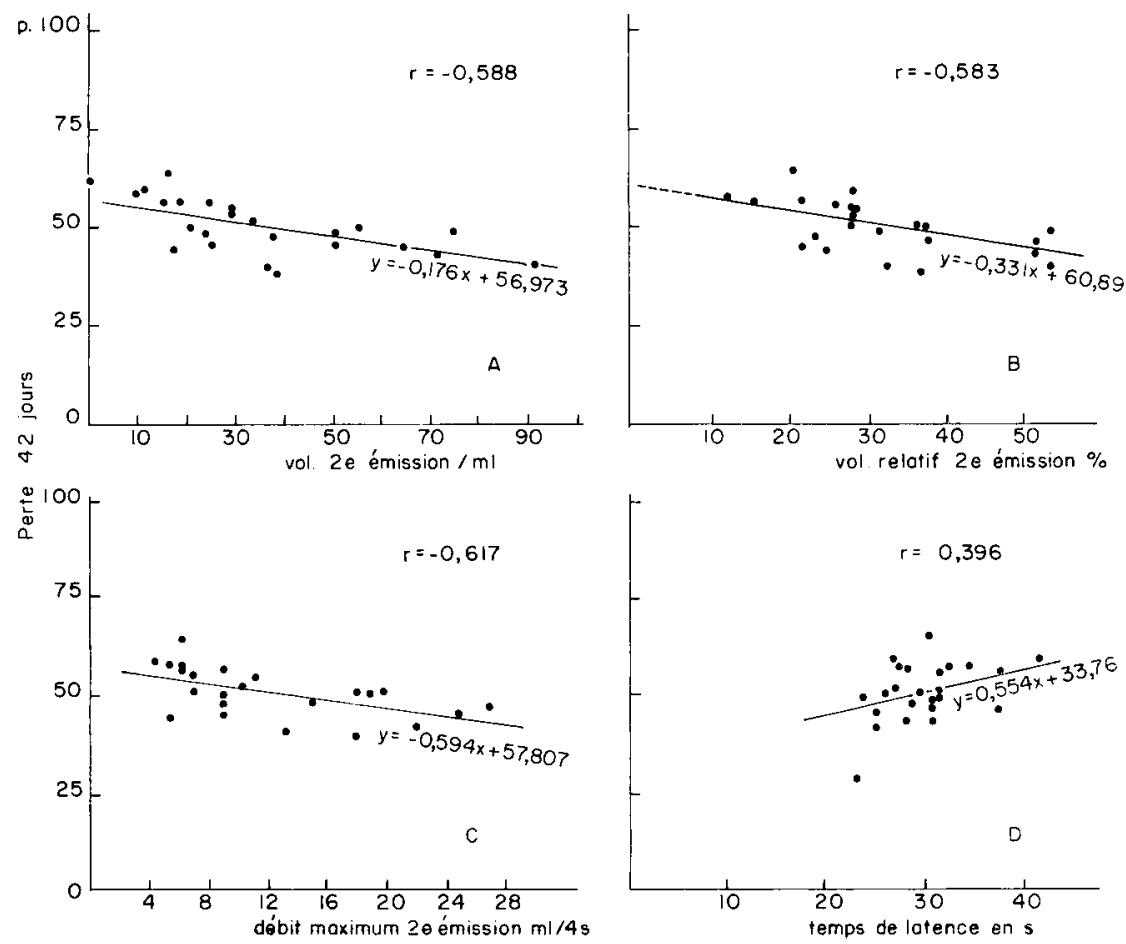

FIG. 4. - Relation enire la perle sur 42 jours el quelpues crileres d'aptilude a la traile
a) Volume absolu de la $2^{\mathrm{c}}$ ćmission
b) Volume relatif de la $2^{\mathrm{e}}$ úmission
c) Lélsit maximum de la $2^{\text {e émission }}$
d) Ialence du réflexe d'éjection 


\section{IV. - DISCUSSION ET CONCLUSIONS}

Les résultats qui viennent d'être présentés nous amènent à faire les remarques suivantes :

I. Dans 80 p. Ioo des cas, le réflexe d'éjection du lait est absent pendant les Io premiers jours de traite qui suivent le sevrage. La disparition de ce réflexe, qui était probablement présent chez tous les animaux au moment de la tétée, peut sans doute constituer une des causes de la baisse de production laitière que l'on constate alors et qui est identique pour les deux groupes d'animaux.

2. L'apparition de la deuxième émission doit être tenue pour partiellement responsable de la production laitière supérieure constatée ensuite chez les brebis du lot 2 .

Il ne faut toutefois pas exclure l'éventualité d'un effet inhibiteur du sevrage sur la sécrétion proprement dite, car le volume total quotidien de lait recueilli à la machine commence à réaugmenter avant même que le réflexe d'éjection ne soit complètement réinstallé.

3. Malgré tout, la production laitière n'est jamais susceptible de retrouver le niveau qu'elle avait atteint pendant la période d'allaitement. On peut émettre quelques hypothèses concernant ce phénomène :

- la rétention, pendant environ ro jours, d'une quantité importante de lait dans les parties supérieures de la glande a pu suffire à freiner définitivement l'activité sécrétoire des cellules mammaires.

- l'extraction du lait alvéolaire provoquée par la machine n'est jamais, même lorsqu'elle existe, aussi importante que celle provoquée par l'agneau. Il nous faut en effet souligner à ce sujet que la perte constatée sur deux périodes successives de 42 jours est d'autant plus élevée que le réflexe d'éjection nous paraît être faible (corrélation inverse entre cette perte et les critères d'appréciation de la $2^{\mathrm{e}}$ émission).

4. Signalons enfin que dans le cas d'une séparation des jeunes 3 jours après la mise bas (LABUSSIÈRE et RICORDEAU, I 968), la proportion des brebis présentant une $2^{\mathrm{e}}$ émission est beaucoup plus forte ( $80 \mathrm{p}$. I00). De plus, 1'apparition de celle-ci se fait alors fréquemment aux alentours de la $7^{\mathbf{e}}$ ou $8^{\mathbf{e}}$ traite, c'est-à-dire plus rapidement qu'à la suite du sevrage relativement tardif que nous avons pratiqué ici.

On peut ainsi supposer que 42 jours de liaisons affectives entre la mère et son agneau rendent plus délicate et plus longue 1'adaptation ultérieure à la machine à traire.

5. Il semble, d'ailleurs, qu'un comportement maternel élevé accroît de façon notable l'ampleur de la perte immédiate consécutive au sevrage. Celle-ci est en effet d'autant plus importante que la persistance individuelle de chaque brebis en période d'allaitement est forte.

Ceci apporte un argument supplémentaire au point de vue des chercheurs israéliens, qui prétendent que la brebis laitière idéale ne doit pas avoir un sens maternel trop développé si l'on veut la traire facilement (EYAL, Volcani et SHaRAV, I958). 


\section{REMERCIEMENTS}

Nous tenons à remercier Mile Aline Solari, MM. Teissonit̀re, Julien, Gouget et Laxague, ainsi que le personnel de la Ferme expérimentale de Brouëssy dont la collaboration nous a été précieuse pour la réalisation de ce travail.

\section{SUMMARY}

\section{INVESTIGATIONS ON THE DECREASE IN MILK YIELD AT WEANING \\ IN RELATION WITH MACIINE-MILKING EFFICIENCY OF THE EWE}

45 one lamb-suckled Préalpes du Sud ewes were weaned after 42 days and machine-milked for 7 weeks. The milk ejection curves during the latter period allowed us to divide the animals into 2 groupes :

$-z_{3}$ animals with two peaks corresponding to the letdown of milk: $1 \%$ from the $2 \%$ from cisterns and acini (group B),

-22 ewes with one single peak (group A); the reasons for the absence of the second peak are still in abeyance.

There weas no significant difference in lactating yields of $A$ or $B$ ewes during the suckling period. The average decrease in milk yield at weaning was similar for the two groups : $38.7 \mathrm{v} \cdot 36.6$ per cent respectively. It is presumabley due to the lack of ejection reflex and to the inhibition of secretory mechanisms.

As soon as day 4 after weaning, the amount of milk ejected by B ewes was significantly higher than with A ewes. For a long machine-milking period, milk yield is thus lower for single peak patterned ewes ((table I,)

For two peak-patterned ewes, milk yield was all the greater as the ejection reflex is nore efficient, i. e. with greater absolute and relative amounts of milk yielded at second peak, higher milk flow, shorter peak-to-peak delay (table 2, figure 4).

The discrepancy between the last estimated amount of suckled milk and that of the first machine-milking is all the greater as the persistency of each ewe during the suckling period is higher (figure 3).

In both $\mathrm{A}$ and $\mathrm{B}$ ewes, the total amount of machine-milked + hand-stripping milk was lower than the amount of suckled milk. Tentative hypotheses accounting for this result are discussed.

\section{RÉFÉRENCES BIBLIOGRAPHIQUES}

Ciolca N., Pirvulescu S., Tafta V., Geogescu D., I960. Amélioration de la méthode d'estimation de la production laitière des brebis pendant la période d'allaitement. Luc. Sit. Inst. Cerc. Zoolech. (Bucaresi $), 18,3^{69-38 \mathrm{r}}$.

Constantinescu O., Gondos G., 1958. Contrôle de la production laitière des brebis pendant la phase d'allaitement. Ann. Inst. Cerc. Zootech., 15, 459-474.

EdeLman Z., I963. The effects of a few factors on persistency of lactation in Awassy sheeps. M. Sc. Thesis Hebrew University Jerusalem (en hébreu). (Cité par Y. Folman, R. Volcani et R. Eyal, ig66).

Eyal E., Volcani R., Sharav E., 1958. Milk secretion and milking efficiency in sheeps. Hanoked., 40, 7-IO.

FIncI M., I957. The improvement of the Awassy breed of sheeps in Israël. Bull. Res. Counc. of Israët, 6 B, I-I06.

Folman Y., Volcani R., Eval E., I966. Mother off spring relationships in Awassy sheeps. I. The effect of different suckling regimes and time of weaning on the lactation curve and milk yield in dairy flocks. J. Agric. Sci. Camb., 67, 359-368. 
GaAL M., I966. Milk yield of ewes during change over from nursing to milking. Allatlennyessles, 15, $273^{-279}$.

Georglou A., I96o. Sheeps and goat raising in Greece. Congrès F. A. O. FEZ Alhènes (Cité par G. RicorDEAU et R. Denamur, I962).

LABUSSIÈres J., Ig66. Relations entre le niveau de production laitière des brebis et leur aptitude a la traite. $X V I I^{\mathrm{e}}$ Congri's Internat. Laiterie, Munich. Section $\mathrm{A}_{1}, 43-5 \mathrm{I}$.

JABLSSIÈrE J., MARTINeT J., 1964. Description de deux appareils permettant le contrôle automatique des débits de lait au cours de la traite à la machine. l'remiers résultats obtenus chez la Brebis. . Tni. Zootech. 13, $199-212$.

I.ABUssitre J., Ricordeau G., r968. Aptitule des brebis à la traite mécanique. Étude des rariations normales au cours de la lactation. Ann. Zoolech. (à paraître).

Labussière J., Denayur R., M.ARtinet J., ig68. Contrôles physiologiques de l'éjection du lait au cours de la traite mécanique de la Brebis : Effets de la dénervation de la mamelle, de l'anesthésie générale de l'animal et de l'injection préalable d'ocytocine (à paraître).

Markus M., 1962. The effed of presence of the lamb on the course of the laclation and on milh yield of Awassy ewes. M. Sc. Thesis, Hebrew Universily. Jerusalem (en hébred). (Cité par Y. Foluax, R. Volcani et E. EyaL, 1966).

Mikes M., 1965. On the study of the milk production by Merino-sheep kept in Slovakia. Zizit?sut Vyroba, 8, $561-574$.

Ricordeau G., Joccard R., Ijexamur R., 1960. Nesure de la production laitière des brebis pendant la période d'allaitement. Ann. Zoolech., 9, 97-I 20.

Ricordeau G., Denamur R., i962. Production laitière des brebis Préalpes du Sud pendant les phases d'allaitement, de sevrage et cle traite. Ann. Zootech., 11, 5-38.

Scheivacker F., I929. Cité par Ulrich, 1953. Die milch und Saügeleistung von Leinesschafen. Zuchunngskunde, 24, $\mathrm{I} 3 \mathrm{I}$. 